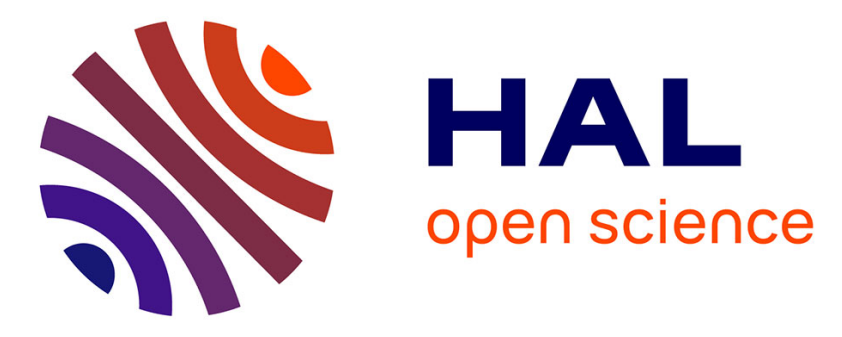

\title{
Large-field high-resolution X-ray microscope for studying laser plasmas
}

\author{
Richard Sauneuf, Jean-Michel Dalmasso, Thierry Jalinaud, Jean-Pierre Le \\ Breton, Daniel Schirmann, Jean-Paul Marioge, Françoise Bridou, Gérard \\ Tissot, Jean-Yves Clotaire
}

\section{To cite this version:}

Richard Sauneuf, Jean-Michel Dalmasso, Thierry Jalinaud, Jean-Pierre Le Breton, Daniel Schirmann, et al.. Large-field high-resolution X-ray microscope for studying laser plasmas. Review of Scientific Instruments, 1997, 68 (9), pp.3412-3420. 10.1063/1.1148302 . hal-00874210

\section{HAL Id: hal-00874210 \\ https://hal-iogs.archives-ouvertes.fr/hal-00874210}

Submitted on 17 Oct 2013

HAL is a multi-disciplinary open access archive for the deposit and dissemination of scientific research documents, whether they are published or not. The documents may come from teaching and research institutions in France or abroad, or from public or private research centers.
L'archive ouverte pluridisciplinaire HAL, est destinée au dépôt et à la diffusion de documents scientifiques de niveau recherche, publiés ou non, émanant des établissements d'enseignement et de recherche français ou étrangers, des laboratoires publics ou privés. 


\title{
Large-field high-resolution x-ray microscope for studying laser plasmas
}

\author{
Richard Sauneuf, Jean-Michel Dalmasso, Thierry Jalinaud, and Jean-Pierre Le Breton \\ Commissariat à l'Energie Atomique (CEA)/Limeil-Valenton, 94195 Villeneuve St-Georges Cedex, \\ France
}

\author{
Daniel Schirmann \\ Commissariat à l'Energie Atomique (CEA)/Bruyères-le-Châtel, BP 12, 91680 Bruyères-le-Châtel, France
}

Jean-Paul Marioge, Françoise Bridou, Gérard Tissot, and Jean-Yves Clotaire

Institut d'Optique Théorique et Appliquée (IOTA), Batiment 503, Centre scientifique d'Orsay, BP 147, 91403 Orsay Cedex, France

(Received 9 September 1996; accepted for publication 13 June 1997)

\begin{abstract}
In 1948, P. Kirkpatrick and A. V. Baez developed an x-ray microscope (energy range about 100 $\mathrm{eV}-10 \mathrm{keV}$ ) composed of two concave spherical mirrors working at grazing incidence. That device, named KB microscope, presents a 3-5 $\mu \mathrm{m}$ resolution within a field having a radius about $100 \mu \mathrm{m}$; outside that field, its resolution lowers rapidly when the object point recedes from the center. The adjunction of two similar mirrors can notably increase the useful field (typically, the resolution can be better than $10 \mu \mathrm{m}$ within a 2-mm-diam field of view), which is necessary for studying laser plasmas. Its main advantage with respect to more simple optics, as the pinhole, is that it can be located far enough from the plasma to avoid any destruction during the shot. We describe such a microscope that we call KBA microscope and present some images of fine metallic grids. Those grids were backlighted by $\mathrm{x}$-ray sources, either a $\mathrm{cw}$ one or a series of laser plasmas from the Octal-Héliotrope facility. Examining the films in detail shows that the experimental results are very close to the theoretical characteristics; hence the interest of this device for the x-ray diagnostics on the future powerful laser facilities. (C) 1997 American Institute of Physics.
\end{abstract}

[S0034-6748(97)02609-9]

\section{INTRODUCTION}

The very hot and very dense plasmas created by laser (more simply called laser plasmas) in order to achieve inertial confinement fusion (ICF) have small dimensions (a few $\mathrm{mm}$, even less than $1 \mathrm{~mm}$ ) and a very short duration (a few ns). Moreover, they include phenomena that are often undesirable such as instabilities the typical dimensions of which are in the order of a few microns. When we want to study these phenomena, we need to have high resolution optical devices able to work in the $\mathrm{x}$-ray range; for our needs, this range spreads from about $100 \mathrm{eV}-10 \mathrm{keV}$ or even a little more.

As a matter of fact, those plasmas emit radiations the spectrum of which presents a maximum within this range; in other respects, when we want to probe those very dense plasmas (x-ray backlighting), we must use $\mathrm{x}$ rays having energies lying in the same range. The desired resolution is in the order of a few microns, if possible about $1 \mu \mathrm{m}$. So the magnification must be $10-50$ to match the resolution of high speed detectors as streak cameras or shutter tubes $(10-20 \mathrm{lp} / \mathrm{mm})$.

When we want to make images with $\mathrm{x}$ rays having energies of about $1 \mathrm{keV}$, the best known optical devices are those which use $\mathrm{x}$-ray glancing reflection on mirrors; the value of the glancing angle $\theta$ is a few degrees, sometimes even lower than $1^{\circ}$.

A device made up of two "crossed" concave spherical mirrors (the incidence planes of the mirrors are perpendicular to one another) was developed in 1948 by Kirkpatrick and Baez $^{1}$ (Fig. 1). This arrangement of mirrors overcomes the strong astigmatism produced by a single concave mirror used at grazing incidence. The rays coming from the object point $A$ and reflected successively by the two mirrors make a pencil which is focused to a small spot, $\left(A^{\prime}\right)$, centered on point $A^{\prime}$. This spot is generally small enough to be considered as a satisfactory image of $A$; so we can say that $A^{\prime}$ is the image of $A$. The broken line $A O_{1} O_{2} A^{\prime}$, composed of the successive mean rays, is called the pseudo axis of the system. Object and image planes, $P$ and $P^{\prime}$ are, respectively, perpendicular to the segments $A O_{1}$ and $O_{2} A^{\prime}$. We also call mean plane of incidence for $M_{1}$ (or more simply $M_{1}$ incidence plane), the plane $I_{1}$ containing the segment $O_{1} O_{2}$ and the normal at $O_{1}$ to the mirror $M_{1} ; M_{2}$ incidence plane is defined in the same way and named $I_{2} . O_{1}$ and $O_{2}$ will be called the apex of their respective mirror.

The previous authors showed that the radius of curvature of both mirrors is very large with respect to the distance between the object and the microscope and that each mirror is equivalent to a cylindrical one having the same radius; so, in order to make their representation easier, we will replace the spherical mirrors by cylindrical ones or even by planes.

Such a device is currently called a KB microscope and is used in many laboratories ${ }^{2}$ to realize images of laser plasmas. However, if the KB microscope can achieve a few micron resolution, this can only be obtained in a rather small field, due to the field obliquity of this device. In order to get a larger field of view, we calculated and realized a similar microscope using four mirrors instead of two; we call that device a KBA microscope (from French "KB amélioré", meaning "improved KB microscope").

This microscope had to satisfy to the following characteristics: 


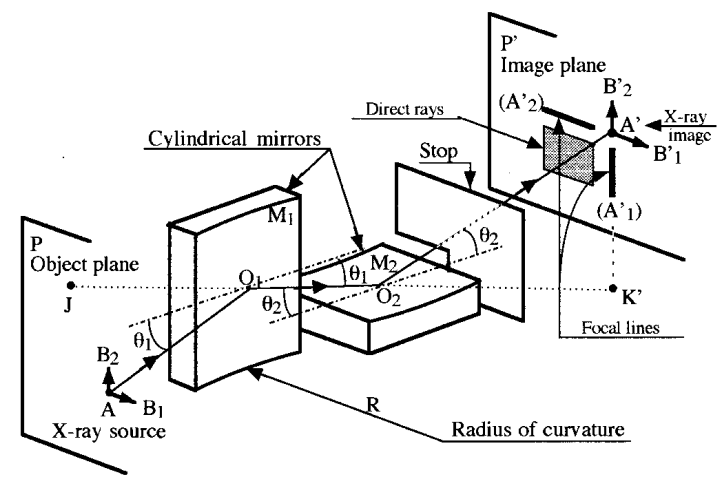

FIG. 1. Schematic arrangement of the Kirkpatrick-Baez microscope which is made of two "crossed" spherical (or cylindrical) mirrors, $M_{1}$ and $M_{2}$, used at grazing incidence. The image of the point $A$ in the object plane $P$ is $A^{\prime}$ in the image plane $P^{\prime}$. The image of $B_{1}$, another point of $P$, not too far from $A$, is not in $P^{\prime}$ (see Fig. 2) but the intersection of the reflected pencil with $P^{\prime}$ is a small spot, nearly circular, centered in $B_{1}^{\prime}$ (the aperture of the incident and reflected pencils is very small). As long as the spot diameter is lower than the desired resolution, we can say that $B_{1}^{\prime}$ is the image of $B_{1}$.

(i) mean magnification: 10 ;

(ii) distance from the object to the microscope entrance: $200 \mathrm{~mm}$;

(iii) distance from the object to the image: about $2.7 \mathrm{~m}$;

(iv) mean grazing angle on each surface: about $2^{\circ}$ (cut-off energy on silica mirrors $\approx 860 \mathrm{eV}$ );

(v) total solid angle of collection: about $4 \mu \mathrm{sr}$;

(vi) resolution at $100 \mathrm{eV}$ better than: $10 \mu \mathrm{m}$ within a field having a radius of $1 \mathrm{~mm}, 30 \mu \mathrm{m}$ within a field having a radius of $2 \mathrm{~mm}$.

We will see later that the characteristics of the completed optical system will be very close to the desired ones.

\section{OPTICAL CONSIDERATIONS}

\section{A. Field obliquity}

A spherical mirror used at grazing incidence gives a focal surface which is strongly inclined to the normal of the principal ray. The inclination of that surface depends on the position of the aperture stop and would be cancelled if a real stop could be placed at a distance of about $2 \mathrm{f} / 3$ from the mirror on the image side. But in the case of the KB microscope, that stop would be difficult to adjust; so the aperture stop is the mirror itself in each plane of focusing.

In one plane of incidence the ray tracing is shown in Fig. 2. Let us consider the image formation of a small object $A B$, situated in this plane and in the object plane, then perpendicular to $A O$. The aperture of the incident and reflected bundles is small so the geometrical aberrations are low and the image $A^{\prime}$ of the point $A$ is a small segment that can be considered as a point if its dimension is lower than the desired resolution.

On the pseudo-axis, we choose $O$ as origin and the light propagation as positive direction. The location of the points $A$ and $A^{\prime}$ is given by the formula:

$$
1 / p^{\prime}-1 / p=1 / f,
$$

where

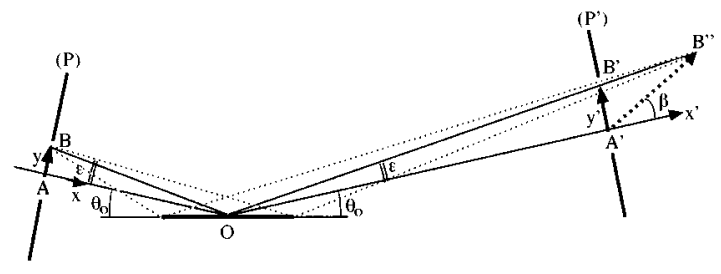

FIG. 2. Image formation of a small object by a spherical mirror working at grazing incidence: the image is affected by the field obliquity aberration. The drawing displays the ray tracing in the incidence plane of the mirror. The focal distance is proportional to the angle $\theta$ between the mirror and the incident ray. For $B$ it is greater than for $A$, so the image of $A B$ is $A^{\prime} B^{\prime \prime}$, with $B^{\prime \prime}$ far outside from $P^{\prime}$ ( $\beta$ is a very small angle). Nevertheless we consider that $A^{\prime} B^{\prime}$, situated in $P^{\prime}$, is the image of $A B$ if $A B$ is small enough.

$$
p=A O, \quad p^{\prime}=O A^{\prime}
$$

and

$$
\begin{aligned}
f & =\left(R \sin \theta_{0}\right) / 2 \\
& \approx R \theta_{0} / 2 \quad \text { (because } \theta_{0} \text { is generally small), }
\end{aligned}
$$

$R$ being positive for a concave mirror and negative for a convex one, $\theta$ being always supposed positive. ( $\theta$ is the general name of the grazing angle between any ray and the mirror; sometimes it may be followed by a subscript to indicate a particular angle as $\theta_{0}$ in the previous relation).

If we consider point $B$, the preceding relations show that its image $B^{\prime \prime}$ is not in the plane $P^{\prime}$ because $\theta$ is different from $\theta_{0}$ when $p$ is fixed; so $p^{\prime}$ has also varied. $A^{\prime} B^{\prime \prime}$ inclination is very important: the angle $\beta$ is about $\theta_{0} p / p^{\prime}$. This is the field obliquity aberration which cannot be corrected in the common KB microscope.

The pencil of rays that converges to $B^{\prime \prime}$ has a very small aperture and its intersection $\left(B^{\prime}\right)$ with the plane $P^{\prime}$ is a little spot centered in $B^{\prime}$. As long as the dimension of this spot is lower than the desired resolution, $B^{\prime}$ can be considered as the image of $B$ and the segment of line $A^{\prime} B^{\prime}$ as the image of $A B$. Within this approximation, the magnification given by one mirror is defined by the formula:

$$
G=y^{\prime} / y,
$$

where $y=A B$ and $y^{\prime}=A^{\prime} B^{\prime}$, with their algebraic signs (see Fig. 2).

It can be easily seen that the magnification becomes

$$
G=-p^{\prime} / p \text {. }
$$

In these relations, $p, p^{\prime}$, and $G$ are algebraic values. Afterwards we will often use the same symbols with the meaning of absolute values; the context will then permit one to understand their meaning.

In the KB system (Fig. 1), a small object $A B$ located in the plane $P$ may be considered as a vector having, in $P$, two components $A B_{1}$ and $A B_{2}$, respectively, parallel to the incidence planes $I_{1}$ and $I_{2}$. Then everything works as if the mirror $M_{1}$ acted only on the component $A B_{1}$ to form the image $A^{\prime} B_{1}^{\prime}$ with the magnification $G_{1}$ defined in (3), the part played by the other mirror being to refocus the diverging rays coming from each point of $A B_{1}$ and situated in incidence planes close to $I_{2}$. By reversing the parts of the two 
a)

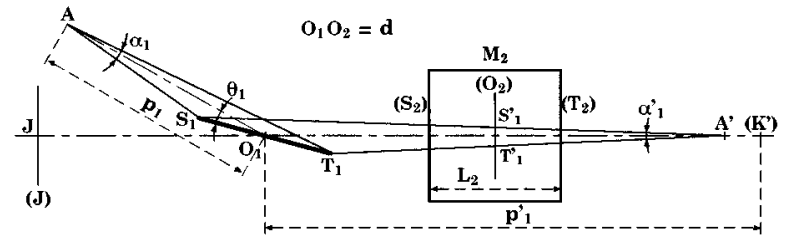

b)

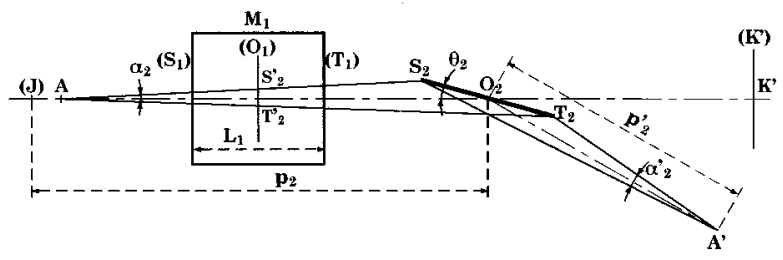

FIG. 3. Aperture of the KB microscope. This schematic drawing displays two views of the optical system (made of two cylindrical mirrors for helping the understanding): (a) a view in $M_{1}$ incidence plane, $I_{1}$, which cuts $M_{1}$ according to the segment $S_{1} T_{1}$ and $M_{2}$ according to the line $\left(O_{2}\right)$; (b) a similar view in $M_{2}$ incidence plane, $I_{2}$. We can see that the incident pencil coming from $A$ is limited by the generating lines $\left(S_{1}\right)$ and $\left(T_{1}\right)$ of $M_{1}$; the aperture of the pencil in $I_{1}$ is $\alpha_{1}$. Within the pencil reflected by $M_{1}$ only a small part can reach $M_{2}$; this part is limited by the generating lines $\left(S_{2}\right)$ and $\left(T_{2}\right)$ of $M_{2}$; the aperture of the pencil in $I_{2}$ is $\alpha_{2}$.

mirrors, $M_{1}$ gives the image $A^{\prime} B_{2}^{\prime}$ with the magnification $G_{2}$. The image $A^{\prime} B^{\prime}$ is then the sum of the two components $A^{\prime} B_{1}^{\prime}$ and $A^{\prime} B_{2}^{\prime}$.

With a good approximation, we can use the following relations:

$$
1 / p_{i}^{\prime}-1 / p_{i}=1 / f_{i} \text { with } f_{i}=R_{i} \theta_{i} / 2
$$

and

$$
G_{i}=-p_{i}^{\prime} / p_{i},
$$

where $i$ can take the values 1 or $2, p_{i}$ and $p_{i}^{\prime}$ representing the algebraic distances along the broken lines joining $O_{i}$ to $A$ and $A^{\prime}$. If we call $d$ the algebraic value of $O_{1} O_{2}$, we can write:

$$
p_{1}^{\prime}=p_{2}^{\prime}+d \quad \text { and } p_{2}=p_{1}-d .
$$

We can see that the magnifications in the two directions are not equal $(G 1>G 2)$ so the image suffers a distortion called anamorphotism; this distortion is not a problem because it is now easy to correct it by digital processing.

\section{B. Aperture of the optical system}

The incident pencil coming from $A$ (as well as the pencil that leaves the microscope and converges to $A^{\prime}$ ) has the form of a pyramid with a nearly rectangular base. To show it more easily, we suppose (Fig. 3) that $M_{1}$ is a cylindrical mirror perpendicular to the plane $I_{1}\left(M_{1}\right.$ incidence plane) and limited by the two generating lines $\left(S_{1}\right)$ and $\left(T_{1}\right)$; the distance $L_{1}$ between those lines is called the length of $M_{1}$. In the same way, $M_{2}$ is a cylindrical mirror perpendicular to the plane $I_{2}\left(M_{2}\right.$ incidence plane $)$ and limited by the two generating lines $\left(S_{2}\right)$ and $\left(T_{2}\right)$; the distance $L_{2}$ between those lines is the length of $M_{2}$.

The rays emitted by $A$ and reflected by $M_{1}$ converge to the line $\left(K^{\prime}\right)$ perpendicular to $I_{1}$ at the point $K^{\prime}$ (symmetrical to $A^{\prime}$ with respect to $M_{2}$ ) and are located inside the

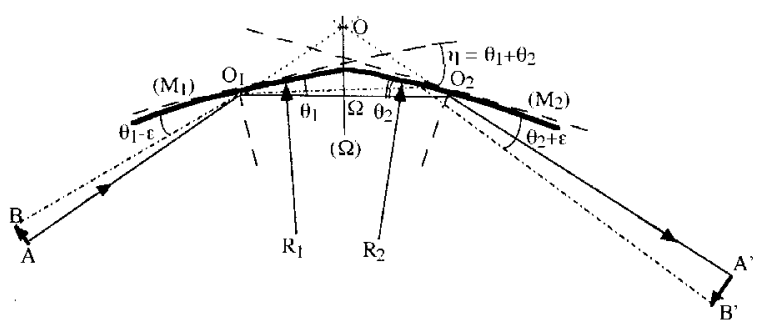

FIG. 4. The association of two "parallel" mirrors (they have the same incidence plane) cancels the field obliquity aberration; we call that association a "bimirror." The drawing displays the ray tracing in the bimirror incidence plane and shows the invariable angle $\eta$ between the mirrors. We see that the angle between $A O_{1}$ and $O_{2} A^{\prime}$ has the same value $(2 \eta)$ than the angle between $B O_{1}$ and $O_{2} B^{\prime}$; it suggests that the focal distance of the system is nearly the same for both points $A$ and $B$ and explains the canceling of the field obliquity.

dihedral angle defined by $A$ and the edges $\left(S_{1}\right)$ and $\left(T_{1}\right)$. In the plane $I_{1}$, represented in Fig. 3(a), we can see that its value is:

$$
\alpha_{1}=\left(L_{1} \sin \theta_{1}\right) / p_{1} \quad\left(\alpha_{1} \text { is a small angle }\right) .
$$

In the same way, the reflected rays are included inside the dihedral angle defined by $\left(K^{\prime}\right)$ and the edges $\left(S_{1}\right)$ and $\left(T_{1}\right)$; its value is

$$
\alpha_{1}^{\prime}=\left(L_{1} \sin \theta_{1}\right) / p_{1}^{\prime} .
$$

Only a part of the previous rays strikes the second mirror. In Fig. 3(b), we see that the useful rays are limited by dihedral angles (values $\alpha_{2}$ and $\alpha_{2}^{\prime}$ ) perpendicular to the previous ones. The intersections of those dihedral angles are two pyramids with rectangular bases.

The aperture angles of the incident pencil $\left(\alpha_{1}\right.$ and $\left.\alpha_{2}\right)$ and those of the emerging one ( $\alpha_{1}^{\prime}$ and $\alpha_{2}^{\prime}$ ) are then, respectively, limited by the mirror lengths $L_{1}$ and $L_{2}$ according to the relations:

$$
\begin{aligned}
& \alpha_{i}=\left(L_{i} \sin \theta_{i}\right) / p_{i}, \\
& \alpha_{i}^{\prime}=\left(L_{i} \sin \theta_{i}\right) / p_{i}^{\prime},
\end{aligned}
$$

which give the following relations:

$$
\alpha_{i}^{\prime} / \alpha_{i}=p_{i} / p_{i}^{\prime}=1 / G_{i} .
$$

As $L_{1} \approx L_{2} \approx L$ and $\theta_{1} \approx \theta_{2} \approx \theta$, we will have:

$$
\alpha_{1} \approx \alpha_{2} \approx \alpha \text { and } \alpha_{1}^{\prime} \approx \alpha_{2}^{\prime} \approx \alpha^{\prime}
$$

so the pyramids will generally be replaced by cones with apex angle $\alpha$ or $\alpha^{\prime}$ (for instance, in the calculation of diffraction).

\section{KBA CONCEPTION}

\section{A. Reduction of field obliquity}

We have just seen that the image $A^{\prime} B^{\prime \prime}$ of a small object $A B$ is strongly inclined because of the variation of $\theta$ from $A$ to $B$ (Fig. 2). The angle between the incident and reflected mean rays is $2 \theta_{0}$ for point $A$ and $2\left(\theta_{0}+\epsilon\right)$ for point $B$. By using a system with two parallel mirrors (both mirrors have the same incidence plane) as suggested by Dyson, ${ }^{3}$ we see (Fig. 4) that the angle between the incident and reflected 


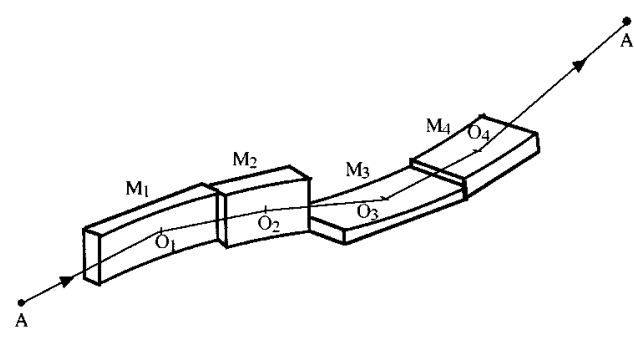

FIG. 5. Schematic arrangement of the KBA microscope. It is composed of two bimirrors which are crossed in order to cancel the astigmatism of a single bimirror.

mean rays is always the same, $2 \eta$, where $\eta=\theta_{1}+\theta_{2}$ is the angle between the two mirrors. We may then think that the focal distance of the system will be nearly the same for $A$ or $B$.

As a matter of fact, by making slight approximations, we can calculate the focal distance of such a system. For practical and theoretical considerations, we will consider the simplest device made up of two parallel mirrors with the same curvature radius $R$ and the same mean grazing angle $\theta$ $=\eta / 2$; moreover they are joined side by side in order to minimize the distance $e=O_{1} O_{2}$. We will call this device a bimirror; its focal distance is practically constant for any point $B$ near to $A$ in the object plane $P$ and its value is

$$
\varphi=R \eta(1+2 e / R \eta) / 8
$$

As $e \ll R \eta$, we can write more simply:

$$
\varphi=R \eta / 8 \text {. }
$$

\section{B. Correction of astigmatism}

It is clear that a bimirror has the same astigmatism as a simple mirror but that shortcoming can be corrected by the use of two crossed bimirrors; this system will then have a high resolution within a rather large field. In order to facilitate the future explanations, we will say that the first bimirror is vertical (its incidence plane being horizontal) and the second one is "horizontal" because its incidence plane is vertical.

This optical system is then composed of four successive mirrors (Fig. 5) and its pseudo-axis is the broken line $\mathrm{AO}_{1} \mathrm{O}_{2} \mathrm{O}_{3} \mathrm{O}_{4} \mathrm{~A}^{\prime}$. Let

$$
\begin{aligned}
& O_{1} O_{2}=e_{1}, \quad O_{3} O_{4}=e_{2}, \quad O_{2} O_{3}=d, \\
& O_{1} A=p_{1}, \quad O_{4} A^{\prime}=p_{4}^{\prime} .
\end{aligned}
$$

This optical system can be defined by desired values of:

(i)

Starting from those data and using relations (5)-(7), we can calculate approximate values of $p_{1}, e_{1}, e_{2}, d$, and $p_{4}^{\prime}$, magnifications $G_{1}$ and $G_{2}$, lengths of mirrors, as well as the focal distances $\varphi_{1}$ and $\varphi_{2}$ of each bimirror. Then we have to choose either $R_{1}=R_{2}$, which facilitate the figuring of the four mirrors which will have the same radius of curvature;

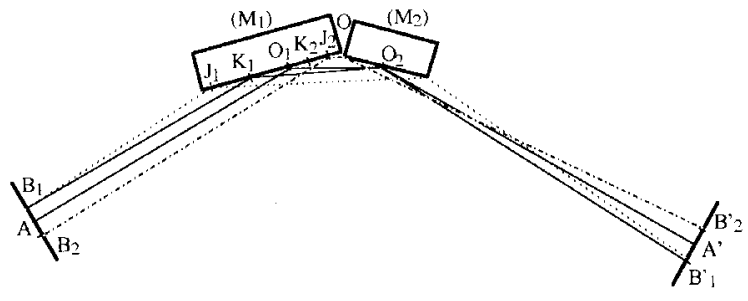

FIG. 6. Field influence in a bimirror. In the KB microscope each mirror plays the part of an aperture stop. In a bimirror, only one mirror must play that part. We chose the second one as the aperture mirror, so the first one (field mirror) had to be longer to allow rays coming from the edges of the desired field $\left(B_{1}\right.$ and $\left.B_{2}\right)$ to strike $M_{2}$ after reflection on $M_{1}$. The theoretical length of $M_{1}$ is $J_{1} J_{2}$ but the mirror is made a little longer.

hence $\eta_{2} \neq \eta_{1}$; or $\eta_{2}=\eta_{1}$, which can give the same spectral response for each mirror and may be necessary when the four mirrors are all together coated with a multilayer; hence $R_{1}$ $\neq R_{2}$.

To calculate a more precise value of the above parameters we have to take into account the field influence which leads to making longer one of the mirrors of each bimirror; we must also use the exact astigmatism relations that include the presence of sagittal and tangential focal lines for each mirror. That leads to an iterative calculation by computer.

\section{Field influence in a bimirror}

As for the $\mathrm{KB}$ microscope, the aperture stop is one mirror of each bimirror; we call it the aperture mirror. The other one, called field mirror, is longer than the aperture one to avoid loosing a part of the pencil when the object point shifts from one edge of the field to the other one (Fig. 6).

Relative location of the two mirrors is then determined as follows:

- The aperture mirror, $M_{2}$, the apex of which is $O_{2}$, is placed behind the field mirror $M_{1}$ and the mean incident ray strikes $M_{1}$ at $O_{1}$. The variation of the field angle is then lower than if the aperture mirror was the first one; calculations show that the resolution variation is also lower.

- The mirrors $M_{1}$ and $M_{2}$ are joined side by side at the point $O$; in order that $M_{2}$ be actually the aperture mirror, it is slightly shifted with respect to $M_{1}$ on which there will be a little "dead" area $\mathrm{OJ}_{2}$ in the vicinity of $M_{2}$. The figure shows that if the shift is low, $O_{1}$ is not at the center of the mirror $M_{1}$; for the ease of assembling, we chose to put $O_{1}$ at the center of the mirror; hence the shift and the dead area are a little more important but still reasonable.

\section{Assembling and adjustment principles}

We imagined a device in which the x-ray mirrors would be held and positioned by steel parts work-finished by a grinding machine. The expected tolerances are $5 \mu \mathrm{m}$ for the essential sizes and $20^{\prime \prime}$ for the angles; we will see that they are sufficient. On the other hand, X-ray mirrors would be figured by optical polishing.

We also had to take into account the way of using and adjusting this microscope which is implemented in a mechanical device (we call it optical unit) that can be fixed on one port of the laser chamber (it will be called output port). 


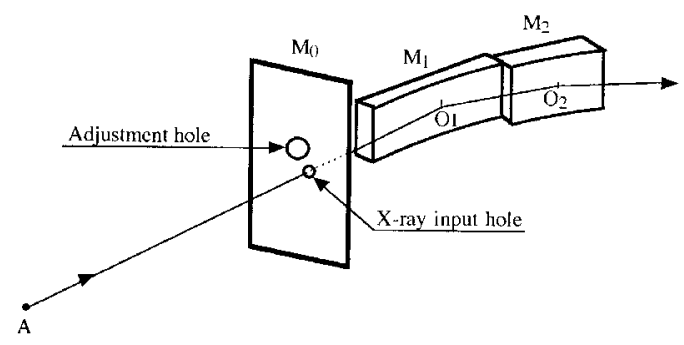

FIG. 7. Adjustment of the microscope. An auxiliary mirror, $M_{0}$, perpendicular to the line $A O_{1}$, is placed in front of the bimirrors and fixed on the base plate that bears them. The useful rays can reach the bimirrors through the x-ray input hole which is centered on $A O_{1}$. For adjusting the microscope, in a laser or test chamber, we use a small diameter laser beam $(\approx 0.5 \mathrm{~mm})$ that simulates the incident $\mathrm{x}$-ray pencil. By tilting the microscope, we make $M_{0}$ perpendicular to that beam. By translating the microscope, the beam is positioned on the center of the adjustment hole, materialized by a pinhole which gives concentric diffraction rings when the beam is well centered; two final translations of known values position it on the center of the input hole.

That device gives the microscope three orthogonal motions of translation under vacuum; a longitudinal one, $X$, along the direction of ray propagation; the other two motions are transverse: $Y$ is horizontal and $Z$ is vertical. Those motions are controlled by step motors and electronic units. At the atmospheric pressure, we can adjust its orientation by means of two knobs.

To obtain the best image, the pseudo-axis must be aligned on the object center. This pseudo-axis is materialized by the normal to the auxiliary plane mirror, $M_{0}$, at the center of a small hole $(\varnothing 2 \mathrm{~mm})$, the $x$-ray input hole, drilled in this mirror (Fig. 7) which is just located in front of $M_{1} ; M_{0}$ protects the x-ray mirrors and the hole in it limits the incident bundle to avoid undesirable illuminations on the detector.

On laser chambers, we can always put a small object at the point that we call the "center" of the chamber. We can also materialize the interesting axis of the chamber by an auxiliary continuous wave laser; its beam is set perpendicular to the plane of the desired port and goes through a pinhole $(\varnothing \approx 0.3 \mathrm{~mm})$ located at the chamber center. The microscope is then tilted so as to make $M_{0}$ perpendicular to the beam. We have now to move the microscope until the beam goes through the center of the input hole but it is not as easy as it seems. We prefer to make the beam go through an auxiliary pinhole $(\varnothing \approx 0.3 \mathrm{~mm})$ bored in a rather thin metallic foil stuck at one end of a small tube which is set in another hole (adjustment hole) in $M_{0}$ (the pinhole is perfectly centered on the axis of the adjustment hole). Behind that pinhole we can see the resulting diffraction pattern and when it is made of well concentric circles, we are sure that the alignment is good with a $\pm 0.05 \mathrm{~mm}$ accuracy. Afterwards we only have to move the microscope by known amounts in the $Y$ and $Z$ directions to be sure that it is well adjusted.

As the focusing depth is rather large $( \pm 1 \mathrm{~mm}$ for an increase of $2 \mu \mathrm{m}$ of the resolution limit in the field center), the distance between the object and the microscope can be monitored by a simple ruler.

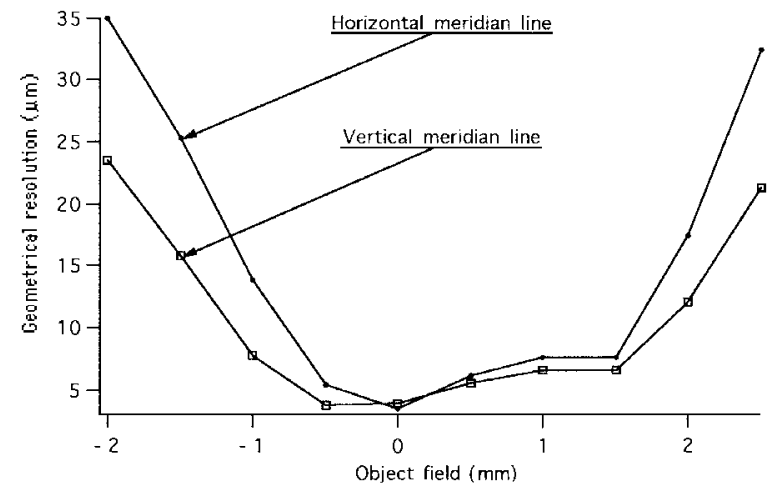

FIG. 8. Geometrical resolution vs object field. That resolution is calculated from the dimensions of spot diagrams given, on the image plane, by rays coming from different points on the horizontal or vertical meridian lines of the object plane; those dimensions are then divided by the corresponding magnifications. Resolution about $7 \mu \mathrm{m}$ is obtained within $2 \mathrm{~mm}$ field.

\section{RESULTS OF COMPUTATION}

\section{A. Radius of curvature and magnification}

In order to facilitate the figuring of mirrors with large radius of curvature and the radius measurement, we decided that the four mirrors would have the same radius $R$. Using the classical astigmatism relations, the results of computation are as follows:

$$
\begin{aligned}
& \theta_{1}=\theta_{2}=2^{\circ} ; \quad \theta_{3}=\theta_{4}=2.3508^{\circ} ; \quad R=23069 \mathrm{~mm} ; \\
& L_{1}=33.70 \mathrm{~mm} ; \quad L_{2}=14.46 \mathrm{~mm} ; \\
& L_{3}=25.02 \mathrm{~mm} ; \quad L_{4}=14.36 \mathrm{~mm} ;
\end{aligned}
$$

Distance from object to image $D=2782 \mathrm{~mm}$.

The system optimization was performed thanks to a ray tracing program developed at the Institut d'Optique Théorique et Appliquée (IOTA) and only based on the geometrical laws of reflection and refraction. We kept $D$ constant and we found that with $R=23130 \mathrm{~mm}$, the images were slightly better and the tolerance on $R$ was broader. In the horizontal plane, the magnification is 11.4 ; it is 9.45 in the vertical plane.

\section{B. Theoretical resolution}

In the image plane, spot diagrams of points regularly distributed in the object field were given by the ray tracing program. They can be assimilated to elliptic spots, the horizontal and vertical dimensions of which are $H^{\prime}$ and $V^{\prime}$. Theoretically, $H^{\prime}$ must be constant when the object point moves on a vertical line; in the same way, $V^{\prime}$ must be constant for a horizontal displacement. Those values are taken as the horizontal and vertical geometrical resolutions in the image plane; in the object plane, the corresponding resolutions, plotted in Fig. 8, are then:

$$
H=H^{\prime} / G_{H} \text { and } V=V^{\prime} / G_{V} \text {. }
$$

We can see that in a field of about $2 \mathrm{~mm}$ in diameter, both resolutions are equal and lower than $7 \mu \mathrm{m}$ (we will call 


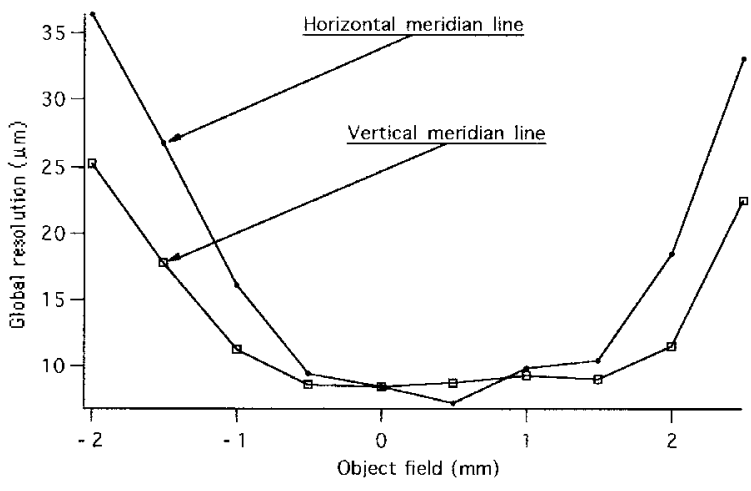

FIG. 9. Global object resolution at $100 \mathrm{eV}$ (estimated). That resolution takes into account the effects of diffraction for $\mathrm{x}$ rays of $100 \mathrm{eV}$ energy. The incident pencil is supposed conical; the diffraction pattern is calculated by the classical formula and added quadratically to the geometrical resolution.

$\Phi_{g}$ this resolution). We call it the "best resolution" field and we can see that it is decentered with respect to the pseudoaxis by about $0.4 \mathrm{~mm}$ in both directions.

For low-energy $x$ rays, the effect of the diffraction is not negligible. On the assumption that the geometrical aberrations are very low, the diffraction would limit the object resolution to $\Phi_{d}$, which can be expressed by the classical relation

$$
\Phi_{d}=1.22 \lambda / \alpha,
$$

where $\alpha$ is the aperture of the incident pencil which is supposedly conical. In our case, $\alpha=2.13 \mathrm{mrad}$ and the maximal value of $\Phi_{d}(7.2 \mu \mathrm{m})$ is reached for $100 \mathrm{eV}$ photons $(124 \AA)$. The length of the aperture mirrors, $M_{2}$ and $M_{4}$, was optimized $^{4}$ for minimizing the global aberration resulting from the superposition of the diffraction and the geometrical aberration. In the $2 \mathrm{~mm}$ field previously defined, $\Phi_{d}$ is of the same order as $\Phi_{g}$ and we will assume that the global resolution is given by the following formula:

$$
\Phi=\left(\Phi_{d}^{2}+\Phi_{g}^{2}\right)^{1 / 2}
$$

Outside that field, we will keep the same formula and give $\Phi_{g}$ the values $H$ or $V$, which will lead to the estimated resolutions plotted in Fig. 9. For higher energies $(>200 \mathrm{eV})$,

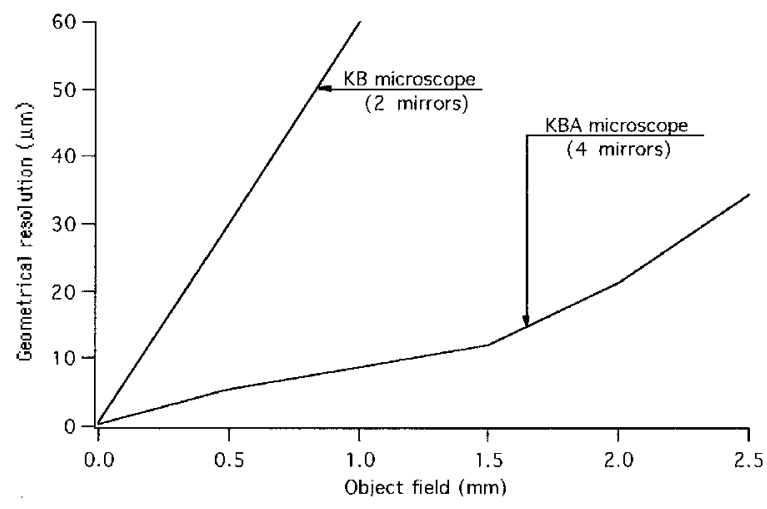

FIG. 10. Approximate comparison of the theoretical resolutions of $\mathrm{KB}$ and KBA microscopes.

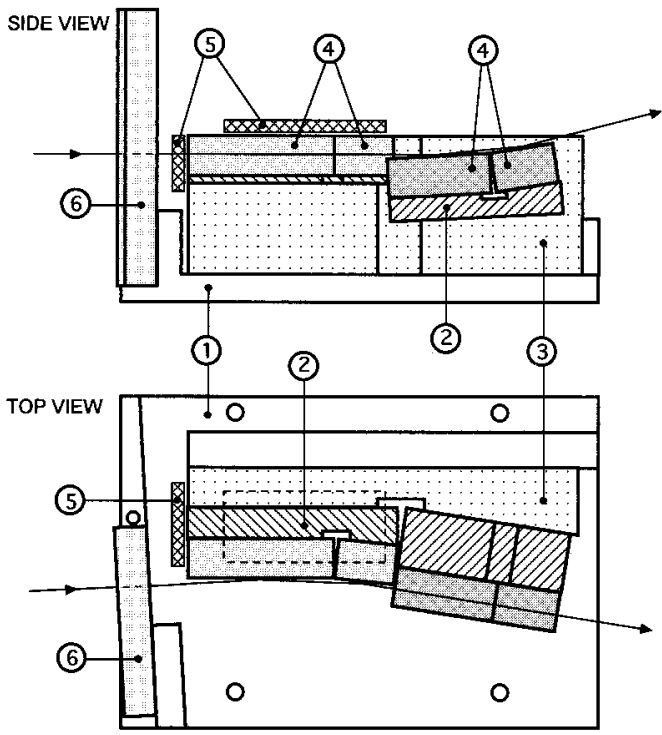

FIG. 11. Drawing of the microscope. Keys: (1) the base plate; (2) the two bearers of bimirror; (3) the assembling part of the two bimirrors; (4) the four x-ray mirrors, $M_{1}-M_{4}$; (5) the stops that are used as reference; (6) the adjustment mirror $M_{0}$. The x-ray mirrors (4) are stuck two by two on the bimirror bearers (2) in order to make the two bimirrors. On each bearer, the two mirrors are located by removable stops (5) which are also used when positioning the bimirrors on the assembling part (3) on which they are screwed (a removable wedge is put under the second bimirror). That part is then screwed on the base plate (1) which bears the adjustment mirror (6).

the diffraction can be considered as negligible. In Fig. 10, we compare the resolutions achieved by two or four mirror optics versus object field.

\section{Tolerances}

The accuracy of the radius of curvature measurement is about $\pm 20 \mathrm{~mm}$ for a 100 -mm-diam mirror. The four mirrors of the microscope are cut up in this mirror and we admit that, thanks to a certain number of previous precautions, their radius is not modified by the process. Taking centering and edging accuracy into account, we took as limits $\pm 20^{\prime \prime}$ for the angles and $\pm 5 \mu \mathrm{m}$ for the essential sizes (for the other sizes we took \pm 0.05 or $\pm 0.5 \mathrm{~mm}$ according to the case) of the steel parts. Then we calculated the effect of such variations on the size of the image spots. The most important parameters were the angles and their variations led to a size increase of $8 \%$ to $9 \%$, which is not negligible. We then had to take care of the tolerances on the other parameters.

\section{MAKING OF THE FOUR-MIRROR MICROSCOPE}

The complexity of this optical system conducted us to choose the principle of a mounting without adjustment. The accuracy of the angular and lateral positioning is obtained by a perfect respect of tolerances for the optical and mechanical parts at each step of the fabrication process.

Figure 11 shows the drawing of the microscope; the caption explains how the different parts are mounted on each other. Except for the x-ray mirrors which are made of silica and cemented on their bearers, all the other parts are made of steel and fastened together by screws; they are located one compared to another due to stops and wedges, some of the stops being used when sticking the mirrors on their bearers. 


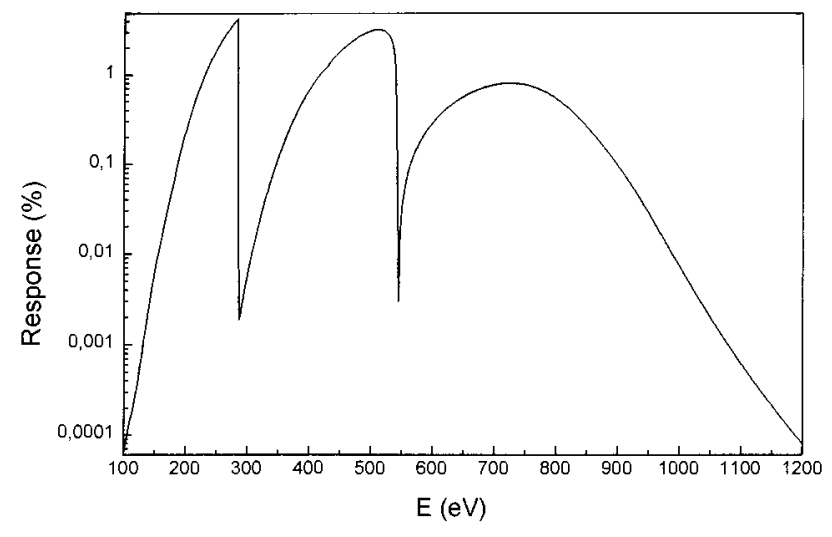

FIG. 12. Spectral response of the microscope associated with a filter (1.5 $\mu \mathrm{m}$ mylar $+0.2 \mu \mathrm{m} \mathrm{Al})$. The curve was computed following the formulas and data given by Henke in Ref. 5; it displays the absorption of the mylar foil due to carbon ( $K$ edge at $284 \mathrm{eV}$ ) and oxygen ( $K$ edge at $532 \mathrm{eV}$ ), added to the reflectivity lack of silica about $532 \mathrm{eV}$ (oxygen).

The front mirror $M_{0}$, which allows the microscope adjustment in the chamber, is also a steel part and its front is optically polished.

The x-ray mirrors which have the same radius of curvature are rectangular parallelepipeds cut up in a silica mirror of $100 \mathrm{~mm}$ in diameter. All their sides are optically polished; the side which will reflect the $\mathrm{x}$ rays is spherical and the tangent plane in its center is parallel to the opposite side with an accuracy much better than $20^{\prime \prime}$. Their widths and thicknesses are nearly the same $(\approx 1 \mathrm{~cm})$; on the contrary, their lengths are very different as indicated in paragraph 4.1. Length and width tolerances are $\pm 0.05 \mathrm{~mm}$ but we have foreseen that the thickness might be adjusted with an accuracy of $1 \mu \mathrm{m}$ with regard to the real measures of their bearers.

\section{EXPERIMENTAL RESULTS}

In Sec. III D, we examined the operating conditions of the microscope in a laser chamber due to the optical unit in which it is implemented. As the photon energy of the $\mathrm{x}$ rays reflected by the mirrors is low $(<800 \mathrm{eV})$, they must propagate under vacuum. An isolating valve is fixed to the output side of the optical unit; the detector [film, charge-coupled device (CCD), camera,...] is connected to that valve by a 2-m-long tube equipped with vacuum pumps and gauges, which allows easy removal of the film after each exposure.

As the detector is generally sensitive to visible and UV radiations emitted by plasmas or even by the filament of classical x-ray sources, it is protected by a filter made of a thin mylar foil coated by $0.2 \mu \mathrm{m}$ of aluminum; the spectral response of the microscope equipped with such a filter is displayed in Fig. 12. We can see that its best response is situated in the lower part of the $\mathrm{X}$-ray range.

\section{A. Tests with the Henke source}

Before mounting the microscope on a laser chamber we wanted to test it on a $\mathrm{cw} \mathrm{x}$-ray source. For that purpose, we dispose of a Henke ${ }^{5}$ source. The optical unit is fixed to a small chamber that simulates a laser one; this chamber is joined to the source by a part in which are implemented the

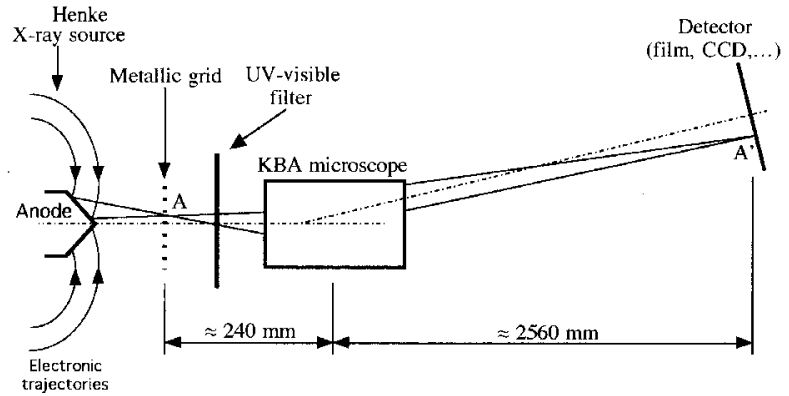

FIG. 13. Schematic arrangement for testing the microscope with the Henke source. The x-rays are emitted by the two front sides of the hexagonal anode; those surfaces are struck by electrons which follow semicircular trajectories from a filament placed behind the anode. So the atoms of tungsten evaporated from the filament cannot directly reach the emitting zone which stay clean longer than in the classical sources. That zone is large enough (about $10 \times 14 \mathrm{~mm}$ ) to backlight the metallic grid over the desired field (about $4 \mathrm{~mm}$ in diameter).

UV filter and the metallic grid which plays the part of a resolution pattern (Fig. 13). As the anode cannot be placed very close to the grid, we need an emitting zone a little larger than the object field; that is the case with the Henke source the emitting zone of which is about $14 \mathrm{~mm}$ high by $10 \mathrm{~mm}$ wide; moreover its brightness is roughly uniform except for a black narrow strip corresponding to the anode edge (Fig. 14).

The images were recorded on Kodak SB2 film. Although it is not very sensitive to low-energy $\mathrm{x}$ rays, it is very convenient and more practical than special films. The source was operated at $12 \mathrm{kV}$ with an electronic current of $100 \mathrm{~mA}$ and the time exposures were about $2 \mathrm{~h}$ each because of the very small amount of $x$-ray power available in the useful part of the spectrum. Figure 14 shows the image of a $25.4 \mu \mathrm{m}$ grid (the period of the grid is $25.4 \mu \mathrm{m}$ in both directions) supported by a 70 mesh grid (period $=363 \mu \mathrm{m}$ ); its useful area is limited by a 4-mm-diam hole in its bearer. On the film we can see that the image of the fine grid is very good nearly all over the field. Figure 15 shows an enlargement of small zone of a $12.7 \mu \mathrm{m}$ grid image: the resolution is still good but

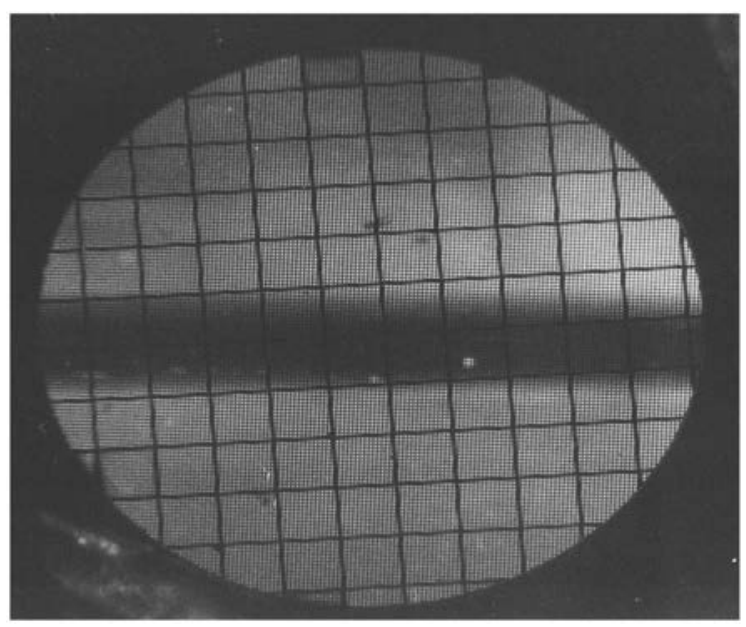

FIG. 14. Image of a $25.4 \mu \mathrm{m}$ grid. That grid is supported by a 70 mesh grid (363 $\mu \mathrm{m}$ period) placed on a hole of $4 \mathrm{~mm}$ in diameter. We can see that the resolution is better than $25 \mu \mathrm{m}$ nearly all over the field. The black strip in the center is due to the lack of $x$ rays from the anode edge. 


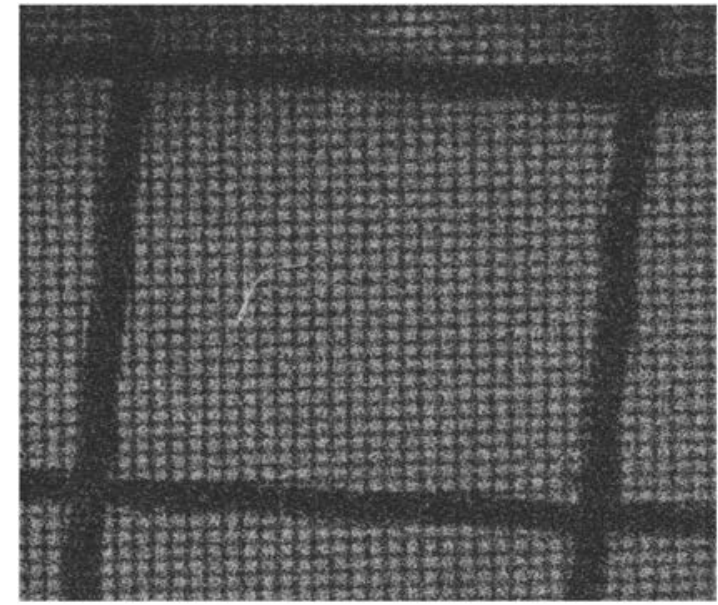

FIG. 15. Image of a $12.7 \mu \mathrm{m}$ grid. This grid is mounted as the previous one. Here we see an enlargement corresponding to one mesh of the supporting grid. The resolution is good but the whole photograph shows that the field is limited to about $2 \mathrm{~mm}$

it is limited to an area corresponding to an object field of 2 $\mathrm{mm}$ in diameter which is decentered by about $0.4 \mathrm{~mm}$ with respect to the center of the 4-mm-diam hole as it was expected in Fig. 8. For lack of finer grids, we could not display the $5 \mu \mathrm{m}$ theoretical resolution in the field center.

The locations of grid and film with respect to the microscope were the theoretical ones. By varying the distance between the $12.7 \mu \mathrm{m}$ grid and the microscope, we were at the best focus and variations of $\pm 2 \mathrm{~mm}$ were necessary to see a loss of resolution at the edge of the $2 \mathrm{~mm}$ field.
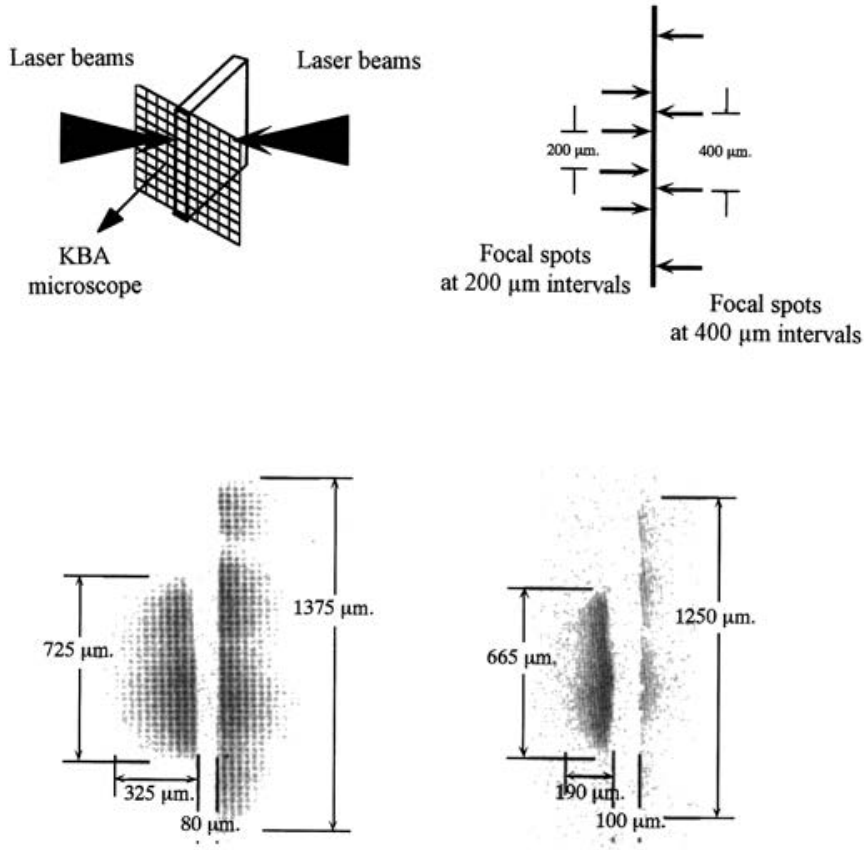

$25.4 \mu \mathrm{m}$ grid

$12.7 \mu \mathrm{m}$ grid

FIG. 16. Resolution tests with a laser plasma x-ray source. The eight laser beams of Octal facility are focused on the two sides of a gold foil, the width of which is about $100 \mu \mathrm{m}$. The energy of each beam is about $40 \mathrm{~J}$ of $3 \omega$ light $(0.35 \mu \mathrm{m})$ deposited in $1 \mathrm{~ns}$ on a $200 \mu \mathrm{m}$ spot $\left(\approx 10^{14} \mathrm{~W} / \mathrm{cm}^{2}\right.$ irradiance). A metallic grid stuck on the edge of that foil is backlighted by the $\mathrm{x}$ rays emitted by the four plasmas created on each side. On the left side, they are rather close to one another and they act as one source as it can be seen on the images of grids displayed under the target drawings; on the contrary, on the right side, they are far enough to be seen as separate sources. The grid images are reconstructed from the data obtained by digitizing the films.
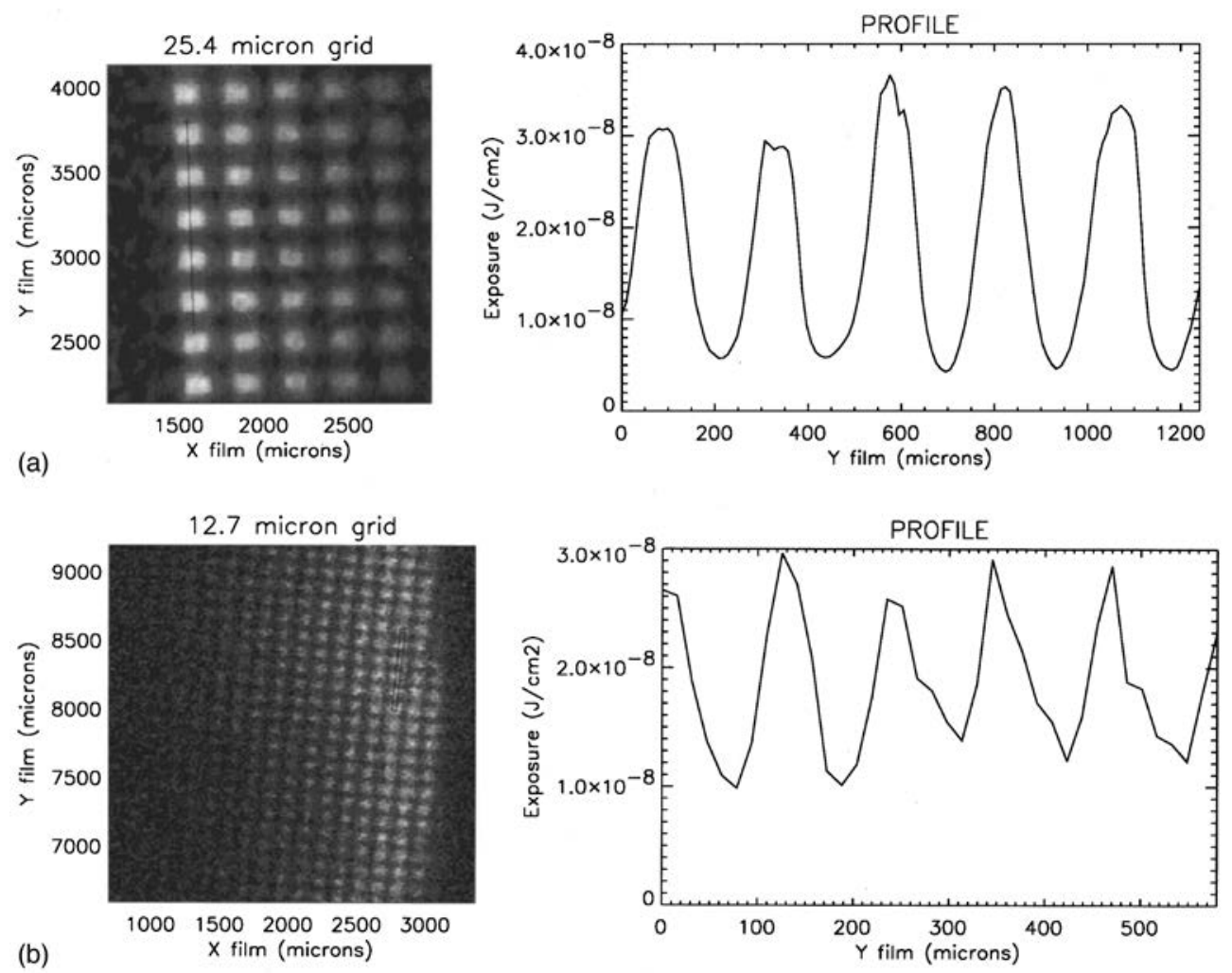

FIG. 17. Exposure profile on the images displayed on the previous figure: (a) $25.4 \mu \mathrm{m}$ grid; (b) $12.7 \mu \mathrm{m}$ grid. In both cases, the contrast is good, (respectively $73 \%$ and $40 \%)$. 


\section{B. Tests on Octal-Héliotrope facility}

Before using the instrument for studying laser plasmas, we checked its spatial resolution in Héliotrope target chamber. Two powerful x-ray sources were created by irradiating each side of a thick plane gold target with four beams of Octal laser; a fine grid was glued on the target extremity as shown in Fig. 16. The energy of each beam was about $40 \mathrm{~J}$ at the wavelength of $0.35 \mu \mathrm{m}$; the pulse duration being $1 \mathrm{~ns}$, the irradiance was on the order of $10^{14} \mathrm{~W} / \mathrm{cm}^{2}$. In order to get a rather large source dimension, we spatially separated the focal spots of the beams in the direction parallel to the grid plane. The intervals were equal to $200 \mu \mathrm{m}$ on one side and $400 \mu \mathrm{m}$ on the other side of the target which gave source lengths on the order of 700 and $1300 \mu \mathrm{m}$, respectively. The sources were located at $3 \mathrm{~mm}$ from the grid which was then backlighted and the KBA microscope gave the image of that grid onto a SB2 film. The protecting filter was made of 1.5 $\mu \mathrm{m}$ mylar coated with $2300 \AA$ of Al. The target, the laser spot positions, and the results are presented in Fig. 16.

The images are reconstructed from the data obtained by digitizing the films. Figure 17 displays enlargements of those images and exposure profiles in the middle of a row of grid holes; for the $25.4 \mu \mathrm{m}$ grid, the contrast is about $73 \%$ and for the $12.7 \mu \mathrm{m}$ one, $40 \%$. The resolution was then convenient to use the KBA microscope for studying any other laser plasmas. Preliminary experiments have been conducted recently and their results will soon be published.

\section{DISCUSSION}

We succeeded in building a grazing incidence $\mathrm{x}$-ray microscope with a high resolution within a large field: about
5-7 $\mu \mathrm{m}$ within a 2-mm-diam field and better than $25-30 \mu \mathrm{m}$ within $4 \mathrm{~mm}$ diameter. Based on one hand on the use of spherical mirrors which may be quite well figured with a very low surface roughness and, on the other hand, on the use of accurate but currently work-finished steel parts, its building is relatively easy and its price is reasonable. Thanks to the low roughness of the mirrors, the scattered light is minimized and the images are well contrasted.

It constitutes an interesting device for laser plasma diagnostics and will be very useful in future experiments conducted with more powerful lasers. For those experiments, it will be necessary to increase its spectral range towards harder $\mathrm{x}$ rays $(1-10 \mathrm{keV})$; that increase could be obtained by lowering the grazing angle (half a degree seems to be a practical limit value) and coating the mirrors with either very dense metal (gold or platinum) or multilayers.

\section{ACKNOWLEDGMENTS}

The authors would like to thank all the people and teams who helped us, especially the IOTA optical workshop which figured the mirrors and assembled the microscope.

${ }^{1}$ P. Kirkpatrick and A. V. Baez, J. Opt. Soc. Am. 38, 766 (1948).

${ }^{2}$ F. Seward, J. Dent, M. Boyle, L. Koppel, T. Harper, P. Stoering, and A. Toor, Rev. Sci. Instrum. 47, 464 (1976).

${ }^{3}$ J. Dyson, Proc. Phys. Soc. London, Sect. B 65, 580 (1952).

${ }^{4}$ R. H. Price and M. J. Boyle, LLNL Laser Program Annual Report 1978 (UCRL-50021-78), pp. 6.14-6.23.

${ }^{5}$ B. L. Henke and M. A. Tester, Advances in X-ray Analysis (Plenum, New York, 1975), Vol. 18, pp. 76-106. 